\title{
Repair of an iatrogenic posterolateral diaphragmatic hernia after pedicled omentoplasty for chronic empyema in an immunocompromised patient: taking down the omentum or not? - A case report
}

\author{
Barbara Donck $^{1 \wedge}$, Marie Cappelle $^{1 \wedge}$, Herbert Decaluwé $e^{1,2}$, Lieven Depypere $^{1,2 \wedge}$ \\ ${ }^{1}$ Department of Thoracic Surgery, University Hospitals Leuven, Leuven, Belgium; ${ }^{2}$ Department of Chronic Diseases and Metabolism (CHROMETA), \\ Laboratory of Respiratory Diseases and Thoracic Surgery (BREATHE), KU Leuven, Leuven, Belgium \\ Correspondence to: Lieven Depypere. Department of Thoracic Surgery, University Hospitals Leuven, Herestraat 49, 3000 Leuven, Belgium. \\ Email: lieven.depypere@uzleuven.be.
}

\begin{abstract}
Iatrogenic diaphragmatic hernias (IDHs) are rare, but the incidence is potentially rising due to the increased implementation of laparoscopy. In case of complications i.e. perforation, bleeding, and/or incarceration, urgent exploration is mandated. However, treatment algorithms lack. We present a case of a 49-year old immunocompromised male patient who developed an IDH with gastric herniation 1 year after performing a pedicled thoracic omentoplasty in the treatment of a recurrent pleurocutaneous fistula. An emergency laparoscopy with 3-trocar approach was performed and the stomach could successfully be reduced. In order to preserve the existing omentoplasty a gastropexy was performed to cover the large diaphragmatic defect $(5 \mathrm{~cm} \times 5 \mathrm{~cm})$. Though, due to persisting postural pain, laparoscopic reintervention with detachment of the gastropexy and omentoplasty took place 6 weeks postoperatively. Diaphragmatic repair was executed using a polypropylene mesh. Further postoperative course was uneventful. One year postoperatively neither chronic pain nor signs of hernia recurrence or empyema are noted. In the repair of IDH caused by a pedicled omentoplasty a laparoscopic approach with mesh repair might be preferred considering previous thoracic surgery and/or infections, overview of the abdominal cavity and diaphragm, and visualization of the pedicle. Nevertheless, the need to preserve the omentoplasty in immunocompromised and other patients should be further investigated, as repair after detachment is technically less complex.
\end{abstract}

Keywords: Iatrogenic diaphragmatic hernia (IDH); gastropexy; pedicled omentoplasty; case report

Received: 06 August 2020; Accepted: 11 April 2021; Published: 25 November 2021.

doi: $10.21037 /$ ccts-20-136

View this article at: http://dx.doi.org/10.21037/ccts-20-136

\section{Introduction}

Acquired diaphragmatic hernias (ADHs) are rare $(<5 \%)$ and occur from penetrating or blunt trauma, or more seldom as a complication of either abdominal or thoracic surgical procedure $(1,2)$. Possibly, the incidence of iatrogenic diaphragmatic hernias (IDHs) is rising due to the increased use of laparoscopy with its long grasping instruments and ultrasonic scissors potentially harming the diaphragm (3). In case of complications of IDH i.e., perforation, bleeding, and/or incarceration, surgical intervention is imperative and urgent. Although some literature is available, no treatment is proven superior. It still remains debatable whether a thoracic or abdominal approach in open or video

^ ORCID: Barbara Donck, 0000-0002-9700-9452; Marie Cappelle, 0000-0001-6149-3206; Herbert Decaluwé, 0000-0002-0877-7717; Lieven Depypere, 0000-0001-8230-5649. 


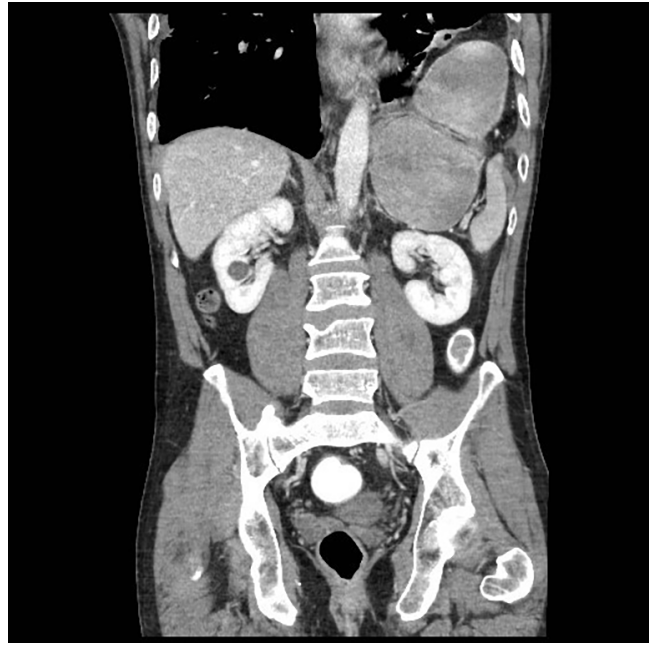

Figure $1 \mathrm{CT}$ of chest and abdomen demonstrating the intrathoracic migration of the greater curvature of the stomach trough a left-sided diaphragmatic defect, previously created for the pedicled omentoplasty.

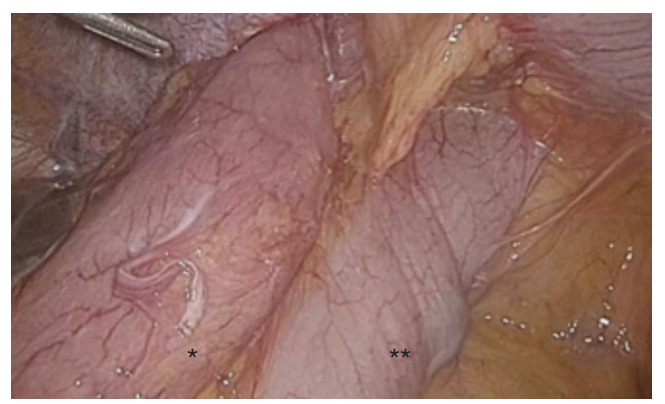

Figure 2 Laparoscopic view of the partial stomach herniation $\left(^{*}\right)$ through the iatrogenic diaphragmatic defect. Note the adherent colon transversum $(* *)$.

assisted fashion needs to be executed and which role mesh reinforcement plays (4).

Pedicled omentoplasties are effective in managing a wide variety of complications in cardiothoracic surgery $(5,6)$. The omentum is a large immune-active and well vascularized organ suitable to fill a large volume defect, can be mobilized easily and is able to absorb wound secretions. Nevertheless, a thoracic omentoplasty necessitates creating an aperture into the thoracic cavity with its consequences coming along.

In this case report, we present a laparoscopic reduction of a partial gastric herniation after pedicled omentoplasty followed by a gastropexy to cover the diaphragmatic defect, but leaving the omentoplasty in place. Due to invalidating postural pain the gastropexy and omentoplasty were taken down six weeks later by laparoscopy, and the hernia defect was closed using a mesh. This case report is presented in accordance with the CARE reporting checklist (available at https://ccts.amegroups.com/article/view/10.21037/ccts-20$136 / \mathrm{rc})$.

\section{Case presentation}

A 49-year-old male presented to the emergency unit with an acute abdomen and complaints of crescendo heartburn, anorexia, and vomitus for 4 days. His past medical history included rheumatoid arthritis, initially treated with methotrexate, nonsteroidal anti-inflammatory drugs and glucocorticoids. As his condition became refractory to the treatment regimen, a therapy-switch was made to certolizumab, a monoclonal antibody specific to tumor necrosis factor alpha. In the following years, the patient presented with recurrent left pleural effusions, pneumonias and empyemas, which were treated by sequential explorative thoracotomies and pleurodeses. Eventually a chronic pleurocutaneous fistula developed. After a year of conservative treatment, a thoracic omentoplasty was created by transferring the omentum, pedicled on the left gastro-epiploic artery, into the thoracic cavity through a 2 by $2 \mathrm{~cm}$ hole in the lateral diaphragm using a hybrid approach i.e. left thoracotomy and laparoscopic approach to successfully repair the fistula. Two months later a laparoscopic adhesiolysis was performed due to gastro-intestinal obstruction with at that time no evidence for an IDH.

At presentation, the patient had mild hypertension $(145 / 98 \mathrm{mmHg})$ and normal heart rate $(72 \mathrm{bpm})$. Abdominal examination showed hyperperistalsis and epigastric rebound tenderness with guarding. Left-sided diminished breath sounds were observed on chest auscultation, oxygen saturation was $97 \%$. Initial blood gas analysis was unremarkable with a normal blood lactate level $(1.14 \mathrm{mmol} / \mathrm{L}$; reference range: 0.5-2.2). Peripheral blood count showed elevated leukocytosis $\left(16 \times 10^{9} / \mathrm{L}\right.$; reference range: $\left.4-10\right)$, and C-reactive protein $(36 \mathrm{mg} / \mathrm{L}$; reference range $\leq 5)$. CT of chest and abdomen revealed an intrathoracic migration of the greater curvature of the stomach through a lateral defect in the left-sided diaphragm (Figure 1). There were no signs of small bowel or colon obstruction, or of compromised vascularization of the omental flap. Abdominal pain persisted after placing a decompressing nasogastric tube; therefore, urgent operative reduction was indicated.

Patient was placed in lithotomy position with an upper body tilt of 45 degrees to the right and both arms alongside the body. One supra-umbilical $12 \mathrm{~mm}$-trocar and 


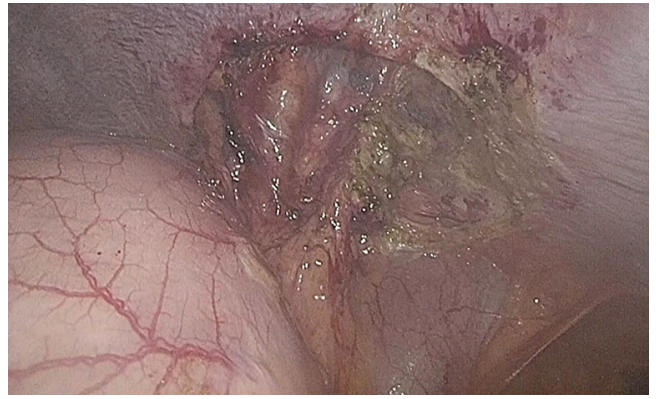

Figure 3 Laparoscopic view after reduction of the stomach exposing the large diaphragmatic defect with viable pedicled omentoplasty in situ.

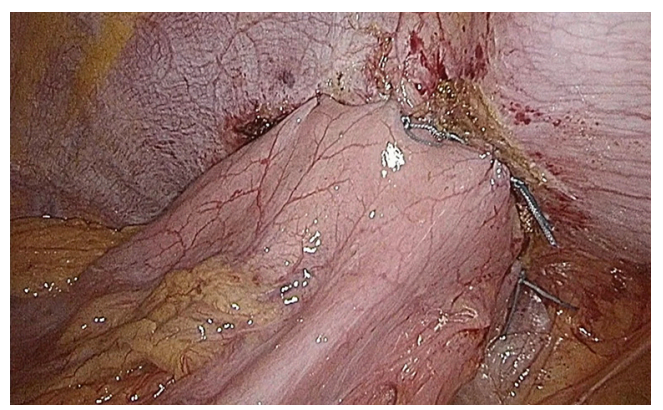

Figure 4 Laparoscopic view of the gastropexy.

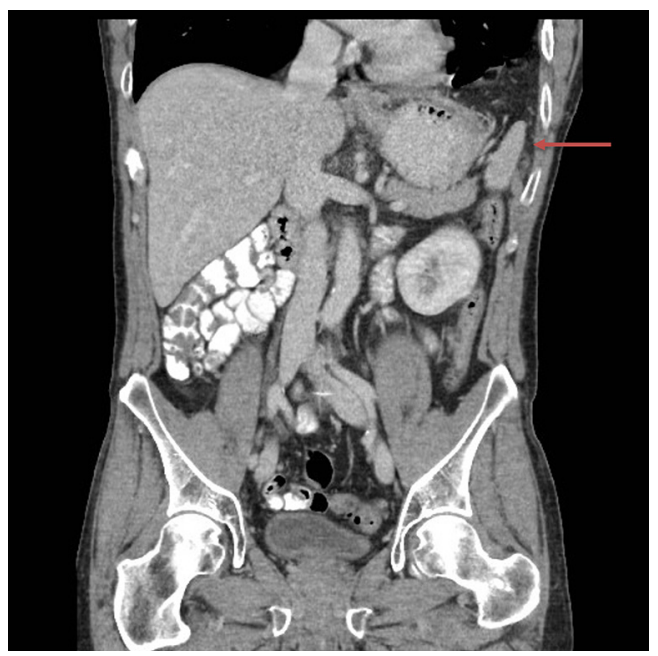

Figure 5 CT of chest and abdomen demonstrating no signs of intestinal perforation or strangulation, and partial splenic herniation (arrow). two $5 \mathrm{~mm}$-trocars in the epigastrium and left flank were inserted. Abdominal inspection showed a partial stomach herniation with adjacent colon transversum (Figure 2). The intrathoracic stomach could be easily reduced by 2 graspers as no intrathoracic adhesions were encountered. Gentle manipulations were used to avoid injuring the pedicle or inducing serosal tears. Hereafter the anterior part of the diaphragm was freed by using scissors and endodissector. After total reduction, a large diaphragmatic defect $(5 \mathrm{~cm} \times 5 \mathrm{~cm})$ was observed with the intact pedicle on the lateral side (Figure 3). The stomach deflated easily when suction on the nasogastric tube was applied. A gastropexy was performed by suturing 6 single polyester stitches (Ethibond ${ }^{\circledR} 0$ ) of the greater curvature of the stomach on to the anterior border of the diaphragmatic defect. No remaining defect could be noted (Figure 4). No postoperative events occurred. Bowel movements were noted on postoperative day 3 after gradually augmenting oral intake. Hospital discharge could take place on postoperative day 4.

Four weeks postoperatively, the patient mentioned good oral intake and normal bowel function. He did note pain upon flexion of the upper body. A postoperative CT showed preservation of the omental flap vasculature and no hernia recurrence. In accordance with the patient a conservative management was opted for.

Six weeks postoperatively, the patient presented at the emergency department with persisting postural pain and vomitus. Vital parameters and blood tests were normal. A thoracic and abdominal film revealed no signs of perforation or strangulation, though a partial splenic herniation was observed (Figure 5). Abdominal pain remained after placing a decompressing nasogastric tube. Therefore, an exploratory laparoscopy was performed after obtaining informed consent. The same laparoscopic set-up was used. Inspection of the abdominal cavity showed no gastric dilatation or herniation, nor signs of strangulation and a well vascularized omentoplasty. A partial splenic herniation was observed without torsion of its vasculature. Due to the persisting pain upon flexion of the upper body the gastropexy was taken down by sharp dissection, and the omentoplasty was detached from the thoracic cavity. The posterior rim of the diaphragmatic defect was dissected free by mobilizing the splenic flexure to create enough space for mesh fixation. A Ventralight ST mesh $(20.3 \mathrm{~cm} \times 25.4 \mathrm{~cm}$, C.R. Bard/ Davol, Warwick, RI, USA) was inserted into the abdomen through the 12-mm trocar and positioned centrally over the defect. Fixation was executed using two circular rows of 


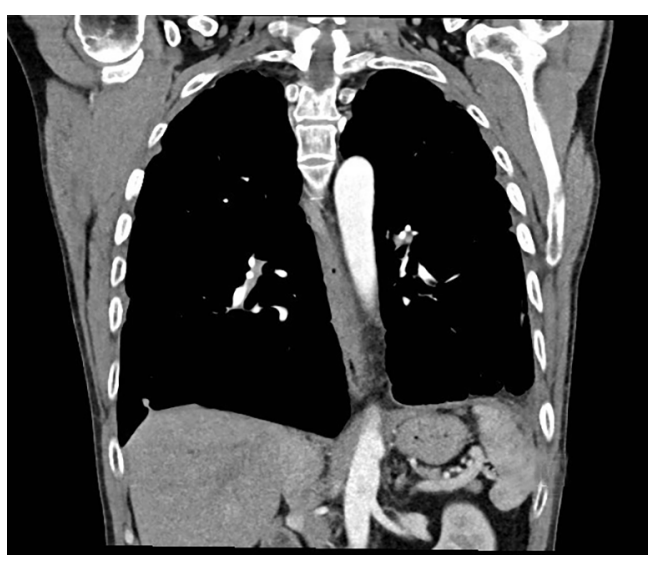

Figure 6 CT of chest and abdomen demonstrating no hernia nor empyema recurrence.

polyester stitches (Ethibond $\left.{ }^{\circledR} 0\right)$. No postoperative events occurred. A thoracic film showed neither hernia recurrence nor other abnormalities after removal of the chest tube on postoperative day 4 , and patient was dismissed on day 8 .

Our patient presented at the emergency department 4 weeks postoperatively with abdominal pain and nausea. CT of the chest and abdomen was negative for hernia recurrence and any other acute abdominal pathology. Spontaneous resolution of his complaints occurred after short observation at our clinic with optimization of analgesia. One week later he was seen at the outpatient clinic in good health. Eighteen months after the last intervention, rheumatologic followup is continued and no chronic pain nor signs of recurrent empyema or hernia are noted (Figure 6). A chronological overview of the complete case is presented on a timeline in Figure 7.

All procedures performed in this study were in accordance with the ethical standards of the institutional and/or national research committee(s) and with the Helsinki Declaration (as revised in 2013). Written informed consent was obtained from the patient for publication of this case report and accompanying images. A copy of the written consent is available for review by the editorial office of this journal.

\section{Discussion}

The first cases of intrathoracic intestinal herniation as complication of a pedicled omentoplasty were described in the early nineties by van Garderen et al. and Massard et al. $(7,8)$. Dividing the omental flap in two parts has been proposed to reduce this complication; one part being transposed into the chest, and a smaller one wrapped around the pedicle to prevent herniation (9). Nevertheless, intrathoracic herniation does occur in this specific setting and treatment algorithms lack. The cornerstone of the management of intrathoracic herniation after thoracic omental flap is to choose a safe approach, consider the need of preserving the omentoplasty, and perform a solid repair to avoid hernia recurrence.

Different surgical approaches in the repair of diaphragmatic hernias have been defended. However, in a large cohort of 38,764 patients, Paul et al. showed that laparoscopic set-up was associated with fewer complications, substantially shorter lengths of stay and earlier functional recovery compared to open thoracic and abdominal procedures (10). Similar outcomes have been reported by others, even in the emergency setting (11-13). A laparoscopic approach, rather than thoracoscopy, may be beneficial due to the overview of other relevant intraabdominal structures and allows the surgeon to examine both diaphragmatic surfaces, the latter more important in traumatic diaphragmatic hernias $(12,14)$. Though, thoracoscopy can be more convenient in right-sided diaphragmatic hernias because of the position of the liver, subsequently obscuring the surgeon's view in laparoscopy (4). As our patient's condition was stable and extensive thoracic adhesions due to previous infections and interventions were awaited, a laparoscopic approach was opted for. Furthermore, laparoscopy offers, particularly in this case, a good view on the omental flap pedicle, and is associated with less surgical trauma, which likely leads to less morbidity in our patient under immunosuppressive therapy.

As the rheumatologic condition of our patient necessitates lifelong immunosuppressive therapy, preservation of the omentoplasty in order to prevent relapse of the pleurocutaneous fistula was considered crucial. A conforming repair therefore becomes more complex. Muysoms et al. described laparoscopic repair of IDH after pedicled omentoplasty developed from experience with other types of hernias (15). They used an intraperitoneal flat mesh without keyhole, thus covering the pedicle, attached to the abdominal wall with sutures and spiral tacks. No intra- or postoperative complications occurred in these case series of 4 patients and no recurrence presented during the first postoperative year. At first, we decided to avoid the use of this technique, because the pedicle was rather medially oriented and directly pulling on the greater curvature of the stomach, probably leading to inadequate mesh fixation 


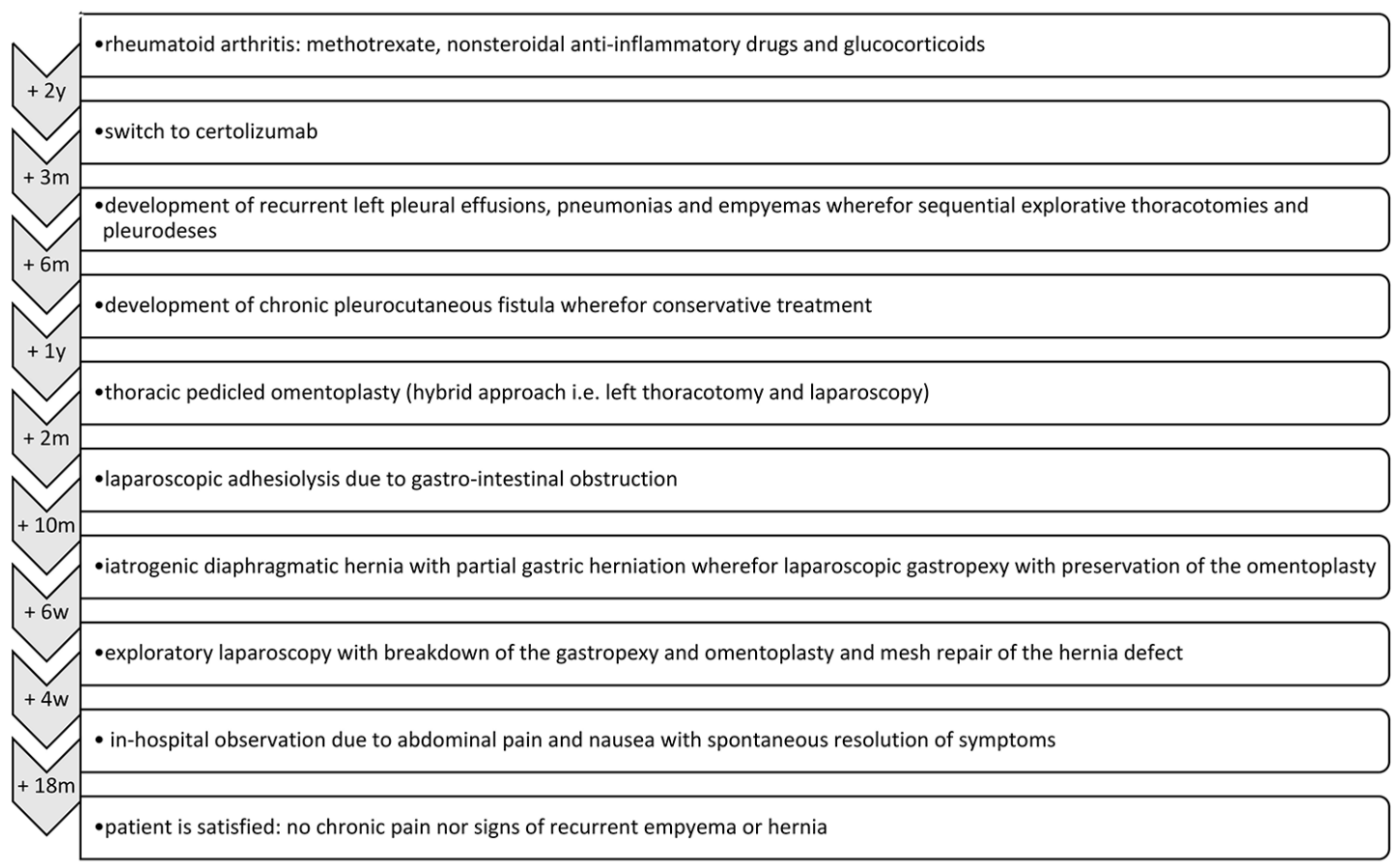

Figure 7 Historical and current information from this episode of care organized as a timeline.

and recurrence of the stomach hernia by sliding under the open end of the mesh. Furthermore, the prolonged immunosuppressive therapy and potentially contaminated environment with strangulated abdominal contents, could lead to a considerable greater risk of mesh infection (4). Therefore, and especially in the light of preserving the omentoplasty, we created a pexy with the use of body's own tissue to cover the major defect.

Laparoscopic anterior gastropexy has been described for the treatment of symptomatic hiatal hernias, especially in high operative risk patients such as the elderly comorbid population $(16,17)$. This minimal invasive approach, with division of the gastrocolic ligament to reduce upward pulling force on the greater curvature, is safe and achieves good short-term results (18). This technique did prevent recurrent gastric herniation, but did not completely cover the diaphragmatic defect as partial splenic herniation occurred (Figure 5). Furthermore, our patient developed postural left hemi-thoracic pain leading to admission at the emergency department 6 weeks postoperatively. Most probable the pain evoked upon flexion of the abdomen is related to the configuration of the gastropexy itself as no recurrent intrathoracic gastric migration was observed at that time (Figure 5), and no other intra-operative abnormality could be withheld. These persisting complaints are likely ischemic in origin due to traction of the omentum on the fixed greater curvature, or due to traction on the vasculature of the spleen caused by its partial herniation, or related to the diaphragmatic sutures put in place during the creation of the gastropexy. In either scenario, the sole solution is taking down the gastropexy.

As we did not believe in the harmony because of the medially oriented pedicle, preserving the omentoplasty and adequate mesh repair, the omentoplasty was taken down too. We considered it to be safe in our patient with at that time a one-year clinical stable condition. We certainly would have been less reluctant to do so if no immunosuppressive therapy was associated. On the other hand, some have stated that the vasculature of the omental flap pedicle becomes independent making it possible to leave the omentoplasty in place after transecting the pedicle and repair the diaphragmatic defect with a prosthesis at the same time (19). This is a promising finding; however more evidence is needed.

The size of the diaphragmatic defect sometimes prohibits a tension-free primary closure, but instead may be achieved by using a mesh, as illustrated in this case. Different types of synthetic and absorbable meshes exist, but evidence favoring a particular type is lacking. Because the diaphragm is under permanent stress due to respiratory and cardiac 
movements, mesh reinforced hernia repair seems to be needed, preferably non-absorbable (4). Biological meshes may be more beneficial when potentially infected areas need to be repaired, as they do not have to be removed when abscess formation occurs (12). Also less problems related to scarring are believed to be seen with the use of biological meshes but experience is limited and the small available data on long-term durability is not that promising, therefore we did not opt for this mesh type in our patient who already underwent several procedures and in whom we wanted to avoid future procedures (12).

Due to inadequate evidence a consensus about optimal mesh fixation technique is also lacking. Definitely, care should be taken when using tacks on the diaphragm in proximity to the pericardium to avoid life-threatening complications (4), with the latter clearly illustrated in a systematic review by Köckerling et al. (20). The question may rise whether tacks should be used at all in this critical anatomical region. Injuries with fixation sutures do occur as well; therefore, carefully positioning the sutures remains the key message. Finally, from the patient perspective: although at first our patient hoped to keep the omentoplasty regarding his extensive medical history, he is still satisfied with the treatment outcome. He felt involved in the decision-making process at all time.

\section{Conclusions}

In the repair of IDH caused by a pedicled omentoplasty a laparoscopic approach with mesh repair might be preferred considering previous thoracic surgery and/or infections, overview of the abdominal cavity and diaphragm, and visualization of the pedicle. Nevertheless, the need to preserve the pedicle of the omentoplasty should be further investigated, as repair after detachment is technically less complex.

\section{Acknowledgments}

Funding: None.

\section{Footnote}

Reporting Checklist: The authors have completed the CARE reporting checklist. Available at https://ccts.amegroups. com/article/view/10.21037/ccts-20-136/rc

Peer Review File: Available at https://ccts.amegroups.com/ article/view/10.21037/ccts-20-136/prf
Conflicts of Interest: All authors have completed the ICMJE uniform disclosure form (available at https://ccts.amegroups. com/article/view/10.21037/ccts-20-136/coif). LD serves as an unpaid editorial board member of Current Challenges in Thoracic Surgery from December 2019 to November 2021. The other authors have no conflicts of interest to declare.

Ethical Statement: The authors are accountable for all aspects of the work in ensuring that questions related to the accuracy or integrity of any part of the work are appropriately investigated and resolved. All procedures performed in this study were in accordance with the ethical standards of the institutional and/or national research committee(s) and with the Helsinki Declaration (as revised in 2013). Written informed consent was obtained from the patient for publication of this case report and accompanying images. A copy of the written consent is available for review by the editorial office of this journal.

Open Access Statement: This is an Open Access article distributed in accordance with the Creative Commons Attribution-NonCommercial-NoDerivs 4.0 International License (CC BY-NC-ND 4.0), which permits the noncommercial replication and distribution of the article with the strict proviso that no changes or edits are made and the original work is properly cited (including links to both the formal publication through the relevant DOI and the license). See: https://creativecommons.org/licenses/by-nc-nd/4.0/.

\section{References}

1. Islam S, Sinha CK, Davenport M. Diaphragmatic Hernia. In: Handbook of pediatric surgery. London: Springer, 2010:69-76.

2. Pan SB, Zhang JB, Zhao BQ, et al. Delayed iatrogenic diaphragmatic hernia after thoracoscopic lobectomy. J Thorac Dis 2016;8:E399-402.

3. Suh Y, Lee JH, Jeon H, et al. Late onset iatrogenic diaphragmatic hernia after laparoscopy-assisted total gastrectomy for gastric cancer. J Gastric Cancer 2012;12:49-52.

4. Machado NO. Laparoscopic repair of Bochdalek diaphragmatic Hernia in Adults. N Am J Med Sci 2016;8:65-74.

5. Kitano M. Omentoplasty in thoracic surgery. Gen Thorac Cardiovasc Surg 2008;56:483-9.

6. Botianu PVH. Current indications for the intrathoracic transposition of the omentum. J Cardiothorac Surg 
2019;14:103.

7. van Garderen JA, Wiggers T, van Geel AN. Complications of the pedicled omentoplasty. Neth J Surg 1991;43:171-4.

8. Massard G, Wilk A, Dumond P, et al. Diaphragmatic hernia complicating omentoplasty after thoracic wall excision. Reflections apropos of 2 cases. Ann Chir Plast Esthet 1992;37:329-32.

9. Tansley P, Kakar S, Withey S. A novel modification of omental transposition to reduce the risk of gastrointestinal herniation into the chest. Plast Reconstr Surg 2006;118:676-80.

10. Paul S, Nasar A, Port JL, et al. Comparative analysis of diaphragmatic hernia repair outcomes using the nationwide inpatient sample database. Arch Surg 2012;147:607-12.

11. Karmali S, McFadden S, Mitchell P, et al. Primary laparoscopic and open repair of paraesophageal hernias: a comparison of short-term outcomes. Dis Esophagus 2008;21:63-8.

12. Pulido J, Reitz S, Gozdanovic S, et al. Laparoscopic repair of chronic traumatic diaphragmatic hernia using biologic mesh with cholecystectomy for intrathoracic gallbladder. JSLS 2011;15:546-9.

13. Hosein S, Carlson T, Flores L, et al. Minimally invasive approach to hiatal hernia repair is superior to open, even in the emergent setting: a large national database analysis.

doi: $10.21037 /$ ccts-20-136

Cite this article as: Donck B, Cappelle M, Decaluwé H, Depypere L. Repair of an iatrogenic posterolateral diaphragmatic hernia after pedicled omentoplasty for chronic empyema in an immunocompromised patient: taking down the omentum or not?-A case report. Curr Chall Thorac Surg 2021;3:42.
Surg Endosc 2021;35:423-8.

14. Oppelt PU, Askevold I, Bender F, et al. MorgagniLarrey diaphragmatic hernia repair in adult patients: a retrospective single-center experience. Hernia 2021;25:479-89.

15. Muysoms FE, Cathenis KKJ, Hamerlijnck RPHM, et al. Laparoscopic repair of iatrogenic diaphragmatic hernias after sternectomy and pedicled omentoplasty. Hernia 2009;13:617-23.

16. El Hajj Moussa WG, Rizk SE, Assaker NC, et al. Large paraesophageal hernia in elderly patients: Two case reports of laparoscopic posterior cruroplasty and anterior gastropexy. Int J Surg Case Rep 2019;65:189-92.

17. Morelli U, Bravetti M, Ronca P, et al. Laparoscopic anterior gastropexy for chronic recurrent gastric volvulus: A case report. J Med Case Rep 2008;2:244.

18. Tanner NC. Chronic and recurrent volvulus of the stomach. With late results of "colonic displacement." Am J Surg 1968;115:505-15.

19. Kolbenschlag J, Hörner C, Sogorski A, et al. Sternal Reconstruction with the Omental Flap--Acute and Late Complications, Predictors of Mortality, and Quality of Life. J Reconstr Microsurg 2018;34:376-82.

20. Köckerling F, Schug-Pass C, Bittner R. A word of caution: never use tacks for mesh fixation to the diaphragm! Surg Endosc 2018;32:3295-302. 